\title{
Prevalence and characterization of Morganella morganii in beef cattle from Sichuan Province, China
}

\author{
bin Tian \\ Sichuan Agricultural University \\ dongjie cai \\ Sichuan Agricultural University \\ xiao liu \\ Sichuan Agricultural University \\ yulong zhang \\ Sichuan Agricultural University \\ jianzhu liu \\ Shandong Agricultural University \\ Mingshu Wang \\ Sichuan Agricultural University \\ Ya Wang \\ Sichuan Agricultural University \\ liping Gou \\ Sichuan Agricultural University \\ zhijun zhong \\ Sichuan Agricultural University \\ Jing Fang \\ Sichuan Agricultural University

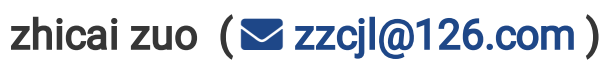 \\ Sichuan Agricultural University
}

\section{Research article}

Keywords: Morganella morganii, cattle, prevalence, antimicrobial susceptibility, pathogenicity

Posted Date: May 11th, 2020

DOI: https://doi.org/10.21203/rs.3.rs-24929/v1 
License: (c) (i) This work is licensed under a Creative Commons Attribution 4.0 International License. Read Full License 


\section{Abstract}

Background Morganella morganii (M. morganii) is a member of the genera Morganella in the Enterobacteriaceae family.

Methods To better understand the prevalence and characterization of M. morganii strains in cattle, 191 nasal swabs samples from beef cattle in Guang'an, Yibin, and Ziyang, Sichuan Province, China were collected. The presence of $M$. morganii in swabs was determined by PCR. Then positive swabs were processed by using bacterial isolation and identification. The $M$. morganii isolates were assayed for antimicrobial susceptibility and pathogenicity using a mouse model.

Results The prevalence of Morgenella morganii in three cities was $10.5 \%(20 / 191)$. Three M. morganii strains (16GA7, 17GA61.2, and 17YB9) were isolated and identified from positive swabs samples. Phylogenetic analysis demonstrated that the 16S rRNA gene of $M$. morganii isolates could be clustered with known Genbank M. morganii strains. All three M. morganii strains were sensitive to penicillin-type and quinolone antibiotics, and were resistant to some cephalosporins, carbapenems, macrolide antibiotics and polymyxin B. Lesions in mice inoculated with $M$. morganii included pulmonary hemorrhage, thickened alveolar walls, and pulmonary inflammatory cell infiltration, although lesions varied by strain.

Conclusion To the best of our knowledge, the present study is the first to report the prevalence of $M$. morganii in beef cattle. Through research on the M. morganii in beef cattle, it is found that mice were susceptible to $M$. morganii isolates, which can cause mice dead. However, the pathogenicity of $M$. morganii isolates on cattle still needs further study.

\section{Background}

Morganella morganii (M. morganii) is a Gram-negative bacterium in the family Enterobacteriaceae that was first isolated in 1906 from a pediatric fecal culture [1]. This bacterium is part of the normal bacterial flora of humans and other animals, and is widely distributed in the environment. M. morganii is an opportunistic pathogen that rarely causes infections, but can be associated with a diverse number of conditions, including sepsis, abscessation, urinary tract infection, cellulitis, diarrhea, and bacteremia [26]. Furthermore, it is capable of causing nosocomial infections and high mortality rates are possible. $M$. morganii infections have been reported in a variety of domestic animals that are associated with humans, including broiler chickens, piglets, rabbits, and dairy calves [7-14]. It is noteworthy that $M$. morganii can live in the oral cavity of animals and thereby cause infections in humans via bites $[3,15-$ 17]. Although the $M$. morganii can rarely cause the occurrence of zoonotic disease, it is noteworthy that M. morganii can spread in weak persons $[18,19]$.

Since the 1970s, M. morganii has been considered an important cause of nosocomial infections in adults, such as urinary tract and wound infections [2]. Some of these infections have had high mortality rates due to $M$. morganil's virulence and increasing drug resistance [20]. M. morganii has also caused 
disease in a variety of animals. M. morganii was isolated from chickens suspected with fowl typhoid or pullorum disease in Nigeria, and important pathologic lesions, including enlarged and congested spleens, enlarged livers congested and friable areas with necrosis, congested lungs, congested, mis-shapened and atrophic ovaries [21]. Large numbers of $M$. morganii were isolated from the pneumonic lesion of a piglet with serofibrinous pleuropneumonia [11]. M. morganii was isolated from the lungs, blood, liver, and blowhole mucosa of a dead dolphin with fibrino-hemorrhagic bronchopneumonia. This death was attributed to septicemia, based on the ecchymoses and petechiae of the spleen, pancreas, forestomach, lungs, visceral peritoneum, and small intestine [7]. In 2018, Li et al. reported a case of M. morganii infection in Holstein calves in Tai'an, Shandong province that originated from lactating cattle with latent infections. In this instance, calves were infected from the drinking milk and the presented clinical signs including depression, poor appetite, paralysis, and feces with white flocs [12]. Little is known about the prevalence of $M$. morganii in Chinese cattle, and this agent was not previously reported in beef cattle.

In the present study, prevalence of $M$. morganii in beef cattle was analyzed, three strains of $M$. morganii were isolated from cow nasal swabs in Guang'an, Yibin and Ziyang, Sichuan Province, China. Furthermore, the biochemical characteristics, drug sensitivity and pathogenicity of these M. morganii isolates were investigated.

\section{Results}

\section{Morgenella morganii prevalence}

Analysis data of the 191 samples showed that the prevalence of Morgenella morganii in three cities was $10.5 \%$ (20/191) \the prevalence in Guang'an city was $18.2 \%$ (14/77) $₫ 7.8 \%$ (5/64) in Yibin, $2 \%(1 / 50)$ in Ziyang. The partial amplified positive PCR products were analyzed by agarose gel electrophoresis (Figure 1). Single DNA positive bands were clearly shown on the gel.

\section{Morgenella morganii culture characteristics and 16S rRNA gene analysis}

Three M. morganii strains (16GA7, 17GA61.2, and 17YB9) were isolated from positive nasal swab samples. Isolates grew as pale pink, translucent round small colonies on MacConkey medium (Figure $2 \mathrm{~A}$ ), and as white, translucent, weakly beta hemolyzed round colonies on blood agar medium (Figure 2B), belonged to gram-negative brevibacterium determined by gram staining (Figure 2C). The 16S rRNA gene of the strains were amplified and sequenced. Through the NCBI BLAST analysis, the similarity between the obtained sequences of isolates and reference M. morganii sequences in Genbank could reach $99 \%$. The 16S rRNA gene sequences of $M$. morganii strains (16GA7, 17GA61.2, and 17YB9) have been deposited in the GenBank database under accession numbers MN807692, MN807693, and MN807694. These strains were phylogenetically clustered with M. morganii strains SQ-1 (GenBank accession number KJ794191.1) and IT-4-1 (GenBank accession number KU570304.1) (Figure 3). The three M. morganii strains had shared biochemical characteristics (Table 2); each strain could metabolize glucose, mannose, ornithine, and phenylalanine, could not metabolize sucrose, maltose, lactose, rhamnose, melibiose, 
arabinose, mannitol, sorbitol, adonitol, inositol, lysine, arginine, esculin, and salicin, and was also H2S test negative. Semi-solid agar puncture test showed that three M. morganii strains had no motility.

\section{Antimicrobial susceptibility testing}

Inhibition diameters and sensitivity for M. morganii strains 16GA7, 17GA61.2, and 17YB9 were shown in Table 3. Strain 16GA7 was sensitive to piperacillin, tetracycline, enrofloxacin, and dose-dependently sensitive to carbenicillin, meropenem, amikacin, and minocycline, and resistant to ampicillin, cephalexin, cefuroxime, imipenem, streptomycin, kanamycin, midecamycin, florfenicol and polymyxin B. Strain 17GA61.2 was sensitive to carbenicillin, piperacillin, amikacin, enrofloxacin, florfenicol, and dosedependently sensitive to cefuroxime, meropenem, streptomycin, and kanamycin, and resistant to ampicillin, cephalexin, imipenem, tetracycline, minocycline, midecamycin and polymyxin B. Strain 17YB9 was sensitive to carbenicillin, kanamycin, tetracycline, minocycline, enrofloxacin, florfenicol, dosedependently sensitive to piperacillin, streptomycin, amikacin, and resistant to ampicillin, cephalexin, cefuroxime, imipenem, meropenem, midecamycin and polymyxin B (Table 3 ).

\section{Mouse virulence}

Mice inoculated with $M$. morganii were depressed, huddled together, had abdominal breathing, and sticky ocular secretions, and moribund mice had open mouth breathing. No clinical abnormalities were evident in control mice. All mice inoculated with strain 16GA7 dead, 3 mice inoculated with strain 17GA61.2 dead, and no mice inoculated with strain 17YB9 dead. Pulmonary hemorrhage was evident in inoculated mice, with no other gross post-mortem pathology evident in other organs. Control mice had no gross pathological abnormalities (Figure 4). Each M. morganii strain was re-isolated from the tissue samples of mice inoculated with the corresponding strain.

Each M. morganii strain presented with similar histopathological findings, although differences were evident (Figure 5). Strain 16GA7 was associated with pulmonary hemorrhage, alveolar wall thickening, pulmonary inflammatory cell infiltration, spleen congestion, hepatic cord disorder, hepatocyte swelling and necrosis, and narrow renal cystic spaces. Strain 17GA61.2 was associated with pulmonary hemorrhage, necrosis, collapsed alveolar structures, pulmonary inflammatory cell infiltration, increased splenic multinuclear macrophages, indistinct splenic white pulp structure, hepatic cords derangement, hepatocyte swelling and necrosis, and narrowing of the renal cystic cavity (Figure 5). Strain 17YB9 was associated with pulmonary hemorrhage, alveolar wall thickening, pulmonary inflammatory cell infiltration, splenic congestion, vacuolar degeneration, hepatic cord derangement, hepatocyte swelling and necrosis, vacuolar degeneration; narrowing of the renal cystic space, and vacuolar degeneration. There were no abnormalities in the cardiomyocytes of the mice infected any of the M. morganii strains. There were no histopathological abnormalities in control mice.

\section{Discussion}


Morganella morganii can be an opportunistic pathogen when the host's immune system is compromised and the agent spreads systemically [22]. Morganella morganii is widely distributed in nature and infections have been reported in fish, amphibians, reptiles, birds, and mammals including humans $[3,23]$. This agent has also been associated with food poisoning in humans [14, 24, 25]. Cattle farming has become a fast-growing industry in China. However, intensive beef cattle farming is facing challenges from infectious diseases. Our findings suggest that bovine $M$. morganii infections are a potential infectious disease concern for the cattle industry.

In our current study, the overall prevalence of Morgenella morganii in Guang'an, Yibin and Ziyang was $10.5 \%(20 / 191)$. This result suggests that the risk of exposure to $M$. morganii in cattle is high in Sichuan. We isolated three M. morganii strains 16GA7, 17GA61.2 and 17YB9 from M. morganii positive nasal swabs collected from beef cattle farms in Sichuan. According to the medical history investigation, these cattle had a history of long-distance transportation shortly prior to disease onset. Transportation-related stressors and may have compromised immunity in these animals, and thereby favoring development of opportunistic M. morganii infections. A dolphin died from septicemia caused by $M$. morganii infection, and transport stress and introduction to a new facility were also considered to be the most likely risk factors [7].

All three M. morganii isolates were sensitive to quinolone and some penicillins-type antibiotics, and resistant to some cephalosporins, carbapenems, macrolide antibiotics and polymyxin B. The spectrum of antibiotics for which $M$. morganii has apparent resistance raises concerns for this agent becoming a multidrug-resistant or extensively drug-resistant zoonosis. A pediatric patient with sepsis was reported to have a $M$. morganii isolate that had NDM-1 and cephalosporinases, although this patient was managed successfully with a combination of aztreonam and ceftazidime-avibactam [26]. A Chinese cobra (Naja naja atra) isolate of M. morganii was reportedly susceptible to piperacillin [23]. Morganella morganii isolated from cattle experiencing high mortality was sensitive to streptomycin, imipenem, aztreonam, and cefoperazone [12]. Taiwanese patients had $M$. morganii isolates with resistance to first-generation cephalosporins and ampicillin-clavulanate [2]. Based on our results, we recommended using enrofloxacin or amikacin for treatment. Differences between our results and previous $M$. morganii studies drug resistance studies $[2,3,12,26,27]$ may be caused by differences between individual strains and environment, including the application history of antibiotic use.

The virulence of M. morganii strains 16GA7, 17GA61.2 and 17YB9 differed, as did the pathology associated with each strain. Among these three M. morganii strains, 16GA7 had the strongest virulence, followed by 17GA61.2, and with 17YB9 having the least virulence. Pathology documented in infected mice included pulmonary hemorrhage, thickened alveolar walls, and pulmonary inflammatory cell infiltration, as well as varying degrees of splenic, hepatic, and renal lesions. These results differ from previous results for sdta1-sdta 5 M. morganii isolates that were associated with hepatic and renal necrosis, hepatic nodules, and renal pelvis hemorrhage with the absence of pulmonary hemorrhage [12]. Comparative genomic analyses demonstrate that $M$. morganii pathogenesis varies with evolution of the agent's virulence [28]. Hosts are also likely more susceptible to M. morganii infection when they are 
immunocompromised. In short, differences among M. morganii isolates need to be further characterized for an improved understanding of pathogenicity, appropriate diagnostic screening, vaccine development, and human public health control measures.

To the best of our knowledge, this study is the first to report the prevalence of $M$. morganii in cattle, and only one case report about dairy calves previously addressed $M$. morganii risks [12]. Nevertheless, $M$. morganii infections may have existed in cattle for a long term without being diagnosed or misdiagnosed. Therefore, $M$. morganii infections are of concern for the potential to become an emerging disease threat to the Chinese commercial cattle industry. Thus, this study is important for understanding the prevalence and pathogenic characteristics of $M$. morganii, and potential therapeutic antibiotics that may be effective for treating infections.

\section{Conclusion}

In our study, prevalence of $M$. morganii was surveyed and three $M$. morganii strains were isolated from beef cattle nasal swabs. In addition, our drug sensitivity assay results demonstrated that $M$. morganii was resistant to multiple antibiotics including cephalosporins, carbapenems, macrolide antibiotics and polymyxin B. Therefore, the development of appropriate management programs for $M$. morganii is vitally important and urgent for the Chinese commercial cattle industry. It is worth pondering that the risk of $M$. morganii infection in humans through contact with cattle or beef foods cannot be ignored.

\section{Methods}

\section{Clinical sample collection}

From April 2016 to October 2019, nasal swabs samples were collected from Sichuan Province beef cattle farms located in Guang'an ( $n=77)$, Yibin $(n=64)$ and Ziyang $(n=50)$ (Table 1). Samples were collected from cattle with recently developed clinical signs following transport from other provinces. Clinical signs included nasal discharge, cough, and dyspnea.

\section{Prevalence of $M$. morganii determined by PCR}

DNA was extracted from nasal swab sample using a One-tube General Sample DNAup for PCR kit (Sangon Biotech (Shanghai) Co., Ltd.). The specific operation was carried out according to the instructions provided by the manufacturer, and the extracted DNA samples were stored at $-20^{\circ} \mathrm{C}$ until analysis by PCR. The specific PCR primers Mm208F (5'-CTC GCA CCA TCA GAT GAA CCC ATA T-3') and Mm1017R (5'-CAA AGC ATC TCT GCT AAG TTC TCT GGA TG-3') were previously developed by Kim and others for detection of M. morganii based on the 16S rRNA sequence [29]. Primers were synthesized by Sangon Biotech (Shanghai). 2×Master Mix (Chengdu Qingke Zixi biotechnology Co., Ltd.) was used for amplification. M. morganii preserved in our lab was used as positive control in PCR. Pooled PCR products were confirmed by comparing DNA size with a DNA mass ladder (Chengdu Qingke Zixi biotechnology Co., Ltd.) on $1 \%$ agarose (Biowest, Spain) gel stained with ethidium bromide (Chengdu Haoboyou 
biotechnology Co., Ltd.). Gels were placed on Gel Documentation system (Bio-Rad Laboratories) for band visualization and photography.

\section{Isolation and identification of $M$. morganii}

Each M. morganii positive nasal swab sample was placed in Tryptic Soy Broth (TSB) medium (Qingdao Hai Bo Biotechnology Co., Ltd.) for pre-enrichment. After incubation at $37^{\circ} \mathrm{Cfor} 24 \mathrm{~h}$, one loop of each preenriched culture was streak-inoculated on to blood agar and MacConkey plates (Qingdao Hai Bo Biotechnology Co., Ltd.) and incubated at $37^{\circ} \mathrm{C}$ for an additional $24 \mathrm{~h}$. Suspected single colonies were selected for microscopic examination with gram staining. Next, the suspected M. morganii purification cultures were identified using bacterial trace biochemical identification tubes (Hangzhou microbial reagent Co., LTD) and PCR amplification. Primer synthesis and PCR products sequencing were conducted by Sangon Biotech (Shanghai) using a 16S rRNA gene primer (Forward primer 5'-

AGAGTTTGATCCTGGCTCAG-3', Reverse primer 5'-TACGGCTACCTTGTTACGACTT-3'). Phylogenetic analysis was performed based on the 16S rRNA gene sequences available in the GenBank database. Phylogenetic trees were reconstructed using MEGA7.0 software [30], with sequences aligned to $M$. morganii reference strains retrieved from GenBank. Bootstrap values were tested with 1000 replicates using a neighbor-joining algorithm, and evolutionary distances were determined using the Kimura twoparameter model.

\section{Antimicrobial susceptibility testing}

The Kirby-Bauer disk diffusion method was used to determine M. morganii isolate antimicrobial susceptibility [31]. Three M. morganii isolates were tested to 16 antibiotics (ampicillin, carbenicillin, piperacillin, cefalexin, cefuroxime, imipenem, meropenem, streptomycin, kanamycin, amikacin, tetracycline, minocyline, midecamycin, enrofloxacin, florfenicol, and polymyxin B) using the Kirby-Bauer disk diffusion method. Results were interpreted following Clinical and Laboratory Standards Institute document M100-S28 guidelines [31].

\section{Mouse virulence}

4-week-old SPF-Kunming mice $(\mathrm{n}=24$, male, weight range $=18-22 \mathrm{~g})$ were purchased from Chengdu $\mathrm{Da}$ Shuo Laboratory Animal Company (China). Mice were randomly assigned to four treatment groups with six mice in each group. Three treatment groups were inoculated with a unique $M$. morganii strain, and one group was set as control. Inoculations were performed using intraperitoneal injections of a suspension made from M. morganii cultures containing $4 \times 10^{8} \mathrm{CFU} / \mathrm{mL}(0.2 \mathrm{~mL} /$ per mouse) in a sterile common broth medium. Control group mice were intraperitoneally injected with equivalent volumes of sterile common broth medium. All mice were monitored for mortality and clinical signs such as ruffled fur, lethargy and dyspnea for $7 \mathrm{~d}$ post-inoculation. Mouse hearts, livers, spleens, lungs and kidneys were collected aseptically post-mortem and fixed in $4 \%$ paraformaldehyde (Solarbio Life Science) for routine processing and embedded in paraffin. Paraffin-embedded tissue samples were sectioned $(3 \mu \mathrm{m})$ and 
stained with hematoxylin and eosin (H\&E) (Beyotime, Beijing). Organ histopathology of all mice was documented by two pathologists who were blinded to treatments.

\section{Abbreviations}

morganii: Morganella morganii; S: Sensitive; S-DD: dose-dependent sensitivity; R: drug resistance.

\section{Declarations}

\section{Ethics approval and consent to participate}

The samples were collected from beef cattle in Sichuan Province, China. The cattle farm leader gave verbal consent for the sample collections. Because there were no vulnerable populations involved. This study was submitted to and approved by Animal Welfare Committee of Sichuan Agricultural University (Approval Number SYXK2019-187). The samples were collected and handled in accordance with the good animal practices required by the Animal Ethics Procedures and Guidelines of the People's Republic of China. Mice used in this study were handled in strict accordance with the guidelines of the Animal Welfare Committee of Sichuan Agricultural University. Mice were maintained in individual cages in a specific pathogen-free environment with an automatically-controlled 12-h light/dark cycle and free access to food and water for seven days. Then, they were randomly divided into different group. Mice were euthanized using sodium phenobarbital according to American Veterinary Medical Association guidelines (2013, AVMA) [32], and every effort was made to minimize animal suffering.

\section{Consent for publication}

Not applicable.

\section{Availability of data and materials}

The 16S rDNA gene sequences of $3 \mathrm{M}$. morganii isolates (16GA7, 17GA61.2, 17YB9) have been deposited in the GenBank database under accession numbers MN807692, MN807693, and MN807694, were free downloaded for scientific research. The other data information during the current study are available from the corresponding author (Zhicai Zuo, zzcjl@126.com) on reasonable request.

\section{Competing interests}

All authors declared no competing conflict of interest.

\section{Funding}

This work was supported by the Program for the National Key Research and Development Program of China, Grant/Award Number: 2018YFD0501800; Sichuan Science and Technology Program, Grant/Award Number: 2018NZ0002/2019YFQ0012; Sichuan Beef Cattle Innovation Team of National Modern 
Agricultural Industry Technology System, Grant/Award Number: 035Z2036. The funders had no role in the design of the study, collection, analysis, and interpretation of data or in the writing of this manuscript.

\section{Authors' contributions}

BT and DJC analyzed the data and drafted the manuscript. YLZ, XL, YW, LPG conducted part of the experiment. JZL and MSW analyzed part of the data. ZJZ, JF, ZCZ participated in the conception and design the experiments and critically revised the manuscripts. All authors read and approved the final manuscript.

\section{Acknowledgements}

We are grateful to the owners of the cattle farm for giving us the opportunity to sample, and to the cattle breeders who cooperated with our sampling.

\section{Authors' information}

${ }^{1}$ Institute of Preventive Veterinary Medicine, Sichuan Agricultural University, Chengdu 611130, China

${ }^{2}$ Department of Veterinary Clinical Sciences, College of Veterinary Medicine, Sichuan Agricultural University, Chengdu 611130, China

${ }^{3}$ College of Veterinary Medicine, Shandong Agricultural University, Tai'an 271018, China

${ }^{4}$ Ministry of agriculture "Veterinary medicine and veterinary technology" Sichuan scientific experimental observation station, Sichuan Agricultural University, Chengdu 611130, China

\section{References}

1. Morgan HDR. Report XCV. Upon the bacteriology of the summer diarrhoea of infants. Br Med J. 1906;1(2364):908-12.

2. Lin TY, Chan MC, Yang YS, Lee Y, Yeh KM, Lin JC, Chang FY. Clinical manifestations and prognostic factors of Morganella morganii bacteremia. European Journal of Clinical Microbiology Infectious Diseases Official Publication of the European Society of Clinical Microbiology. 2015;34(2):231-6.

3. Liu H, Zhu J, Hu Q, Rao X. Morganella morganii, a non-negligent opportunistic pathogen. International Journal of Infectious Diseases. 2016;50:10-7.

4. Nakajima M, Shirokawa M, Miyakuni Y, Nakano T, Goto H. Giant iliopsoas abscess caused by Morganella Morganii. American Journal of Case Reports. 2017;18:395.

5. Katz LM, Lewis RJ, Borenstein DG. Successful joint arthroplasty following proteus morganii (morganella morganii) septic arthritis: a four-year study. Arthr Rhuem. 2014;30(5):583-5.

6. Salen PN, Eppes S. Morganella morganii: a newly reported, rare cause of neonatal sepsis. Academic Emergency Medicine Official Journal of the Society for Academic Emergency Medicine. 
2014;4(7):711-4.

7. Elfadl AK, Lee SW, Kim JH, Lee KL, Arif Ullah HM, Chung MJ, Ghim SG, Lee EJ, Kim YD, Kim SM. Fatal fibrino-hemorrhagic bronchopneumonia associated with Morganella morganii in a bottlenose dolphin: a case report. Diseases of Aquatic Organisms. 2017;127(1):41-7.

8. Yu EQ, Zhang D, Li DM, Yang Z, Zai XS, Gong JS, Qin AJ, Jin WJ. Isolation,identification and genetic evolution analysis of Morganella morganii from eggs. China Animal Husbandry \& Veterinary Medicine 2013, 8.

9. Zhao Y, Li WJ, Du XB, Chen M, Kang K, Chen YL. Phylogenetic analysis and identification of biological characterization of Morganella Morganii in kangaroo. China Animal Husbandry Veterinary Medicine. 2010;37(3):48-51.

10. Wani SA, Bhat MA. Antibiogram and bacterial flora of pyogenic infections of domestic animals in kashmir valley. Indian Journal of Animal Sciences. 2003;73(4):384-6.

11. Ono M, Namimatsu T, Ohsumi T, Mori M, Okada M, Tamura K. Immunohistopathologic demonstration of pleuropneumonia associated with Morganella morganii in a piglet. Vet Pathol. 2001;38(3):336-9.

12. Li G, Niu X, Yuan S, Liang L, Liu Y, Hu L, Liu J, Cheng Z: Emergence of Morganella morganii subsp. morganii in dairy calves, China. 2018, 7(1).

13. Lei $K$, Zhu NY, Bei YJ, Ding XY, Chen JS. Isolation and identification of pathogenic Morganella morganii isolates from Chinese soft-shelled turtle pelodiscus sinensis. Oceanologia Et Limnologia Sinica. 2013;44(3):722-7.

14. McCarthy S III. WB: Efficacy of electrolyzed oxidizing water against Listeria monocytogenes and Morganella morganii on conveyor belt and raw fish surfaces. Food Control. 2012;24(1-2):214-9.

15. Chen CM, Wu KG, Chen CJ, Wang CM. Bacterial infection in association with snakebite: A 10-year experience in a northern Taiwan medical center. J Microbiol Immunol Infect. 2011;44(6):456-60.

16. Chunyi L, Hsiufen L, Huang FL, Chen PY. Haemorrhagic bullae associated with a chicken scratch. Ann Trop Paediatr. 2009;29(4):309-11.

17. Fischer J, Hille K, Mellmann A, Schaumburg F, Kreienbrock L, Köck R. Low-level antimicrobial resistance of Enterobacteriaceae isolated from the nares of pig-exposed persons. Epidemiology Infection. 2016;144(4):686-90.

18. Di lanni F, Dodi PL, Cabassi CS, Pelizzone I, Sala A, Cavirani S, Parmigiani E, Quintavalla F, Taddei S. Conjunctival flora of clinically normal and diseased turtles and tortoises. BMC Vet Res. 2015;11(1):91.

19. Yacoub A, Janz T, Mojica L, Jones L, Greene JN. A fishmonger's tale: a case report and review of literature. Infectious Diseases in Clinical Practice. 2015;23(5):248-53.

20. Lee IK, Liu JW. Clinical characteristics and risk factors for mortality in Morganella morganii bacteremia. J Microbiol Immunol Infect. 2006;39(4):328-34. 
21. Mamman PH, Kazeem HM, Raji MA, Nok AJ, Kwaga JKP. Preliminary report on isolation of Morganella Morganii from fatal infections of chickens in Kaduna State. Nigeria. 2014;1(6):77-80.

22. Earley B, Sporer KB, Gupta S. Invited review: Relationship between cattle transport, immunity and respiratory disease. Animal. 2016;11(3):486-92.

23. Wang HF, Du LY, Luo J, He HX: Isolation, identification and characterization of Morganella morganii from Naja naja atra in Beijing, China. Cellular and molecular biology (Noisy-le-Grand, France) 2017, 63(7):52.

24. Papadopoulou C, Economou E, Zakas G, Salamoura C, Dontorou C, Apostolou J. Microbiological and pathogenic contaminants of seafood in Greece. J Food Qual. 2007;30(1):28-42.

25. Fernández-No IC, Böhme K, Gallardo JM, Barros-Velázquez J, Calo-Mata P. Differential characterization of biogenic amine-producing bacteria involved in food poisoning using MALDI-TOF mass fingerprinting. Electrophoresis. 2010;31(6):1116-27.

26. Hobson CABS, Fahd M, Baruchel A, Cointe A,Poey N,Jacquier H, Doit C,Monjault A,Tenaillon O: Successful treatment of bacteremia due to NDM-1-producing Morganella morganii with aztreonam and ceftazidime-avibactam combination in a pediatric patient with hematologic malignancy. Antimicrobial agents and chemotherapy 2019, AAC-02463.

27. Schultz E, Barraud O, Madec JY, Haenni M, Doublet B. Multidrug resistance Salmonella genomic island 1 in a Morganella morganii subsp. morganii human clinical isolate from France. Msphere. 2017;2(2):e00118-00117.

28. Chen YT, Peng HL, Shia WC, Hsu FR, Ken CF, Tsao YM, Chen CH, Liu CE, Hsieh MF, Chen HC. Wholegenome sequencing and identification of Morganella morganii KT pathogenicity-related genes. Bmc Genomics. 2012;13(7):1-14.

29. Leary SL, Underwood W, Anthony R, Cartner S, Corey D, Grandin T, Greenacre C, Gwaltney-Bran S, McCrackin M, Meyer R: AVMA guidelines for the euthanasia of animals: 2013 edition. In: 2013: American Veterinary Medical Association Schaumburg, IL; 2013.

30. Kim SH, An H, Wei Cl, Visessanguan W, Pitta TP. Molecular Detection of a Histamine Former, Morganella morganii, in Albacore, Mackerel, Sardine, and a Processing Plant. J Food Sci. 2006;68(2):453-7.

31. Sudhir K, Glen S, Koichiro T: MEGA7: molecular evolutionary genetics analysis version 7.0 for bigger datasets. Molecular Biology \& Evolution 2016, 33(7):1870-1874.

32. M100 Performance Standards for Antimicrobial. In.: Clinical and Laboratory Standas Institute 950 West Valley Road, Suite 2500 \&\#8230.

\section{Tables}

Table 1 The Sample types, clinical syndrome, farm location and number of samples 


\begin{tabular}{lccc}
\hline Sample types & Clinical syndrom & Farm location & Number of samples \\
\hline nasal swabs & respiratory symptoms & Guang'an & 77 \\
nasal swabs & respiratory symptoms & Yibin & 64 \\
nasal swabs & respiratory symptoms & Ziyang & 50 \\
\hline
\end{tabular}

Note: respiratory symptoms including nasal discharge, cough, and dyspnea.

Table 2 Biochemical test results for 16GA7, 17GA61.2, 17YB9 strains of M. morganii

\begin{tabular}{cccc}
\hline Substrate & Results & Substrate & Results \\
& & & \\
\hline Glucose & + & Mannitol & - \\
Sucrose & - & Sorbitol & - \\
Maltose & - & Adonitol & - \\
Lactose & - & Inositol & - \\
Mannose & + & Phenylalanine & + \\
Rhamnose & - & Ornithine & + \\
Melibiose & - & Lysine & - \\
Arabinose & - & Arginine & - \\
Semi-Solid Agar & - & Esculin & - \\
$\mathrm{H}_{2} \mathrm{~S}$ & - & Salicin & - \\
\hline
\end{tabular}

Table 3 Drug sensitivity test results for 16GA7, 17GA61.2, 17YB9 strains of $M$. morganii

\begin{tabular}{ccccccc}
\hline Drugs & \multicolumn{2}{c}{ Inhibition diameters/mm } & \multicolumn{3}{c}{ Sensitivity } \\
& 16 GA7 & 17 GA61.2 & 17 YB9 & 16GA7 & 17 GA61.2 & 17 YB9 \\
\hline Ampicilin & 13.0 & 8.0 & 8.0 & $\mathrm{R}$ & $\mathrm{R}$ & $\mathrm{R}$ \\
Carbenicilin & 19.0 & 26.3 & 32.0 & $\mathrm{~S}-\mathrm{DD}$ & $\mathrm{S}$ & $\mathrm{S}$ \\
Piperacilin & 25.0 & 22.3 & 18.5 & $\mathrm{~S}$ & $\mathrm{~S}$ & $\mathrm{~S}-\mathrm{DD}$ \\
Cephalexin & 8.0 & 8.0 & 8.0 & $\mathrm{R}$ & $\mathrm{R}$ & $\mathrm{R}$ \\
Cefuroxime & 9.0 & 17.0 & 10.5 & $\mathrm{R}$ & $\mathrm{S}-\mathrm{DD}$ & $\mathrm{R}$ \\
Imipenem & 14.0 & 13.5 & 10.0 & $\mathrm{R}$ & $\mathrm{R}$ & $\mathrm{R}$ \\
Meropenem & 15.0 & 16.0 & 14.0 & $\mathrm{~S}-\mathrm{DD}$ & $\mathrm{S}-\mathrm{DD}$ & $\mathrm{R}$ \\
Streptomycin & 12.0 & 16.0 & 17.0 & $\mathrm{R}$ & $\mathrm{S}-\mathrm{DD}$ & $\mathrm{S}-\mathrm{DD}$ \\
Kanamycin & 11.0 & 19.8 & 22.0 & $\mathrm{R}$ & $\mathrm{S}-\mathrm{DD}$ & $\mathrm{S}$ \\
Amikacin & 15.0 & 20.5 & 19.0 & $\mathrm{~S}-\mathrm{DD}$ & $\mathrm{S}$ & $\mathrm{S}-\mathrm{DD}$ \\
Tetracycline & 23.0 & 11.0 & 21.8 & $\mathrm{~S}$ & $\mathrm{R}$ & $\mathrm{S}$ \\
Minocycline & 18.0 & 9.5 & 20.7 & $\mathrm{~S}-\mathrm{DD}$ & $\mathrm{R}$ & $\mathrm{S}$ \\
Midecamycin & 8.0 & 8.0 & 8.0 & $\mathrm{R}$ & $\mathrm{R}$ & $\mathrm{R}$ \\
Enrofloxacin & 20.0 & 22.8 & 23.0 & $\mathrm{~S}$ & $\mathrm{~S}$ & $\mathrm{~S}$ \\
Florfenicol & 8.0 & 25.3 & 24.3 & $\mathrm{R}$ & $\mathrm{S}$ & $\mathrm{S}$ \\
PolymyxinB & 8.0 & 8.0 & 8.0 & $\mathrm{R}$ & $\mathrm{R}$ & $\mathrm{R}$ \\
\hline & & & & & &
\end{tabular}




\section{Figures}

\section{0 bp}

\section{0 bp $750 \mathrm{bp}$ 500 bp}

\section{0 bp} 100 bp

\section{Figure 1}

The partial amplified positive PCR products analyzed by agarose gel electrophoresis. M: DNA Marker, P: positive control, 1-4: samples PCR products, 5: negative control. 

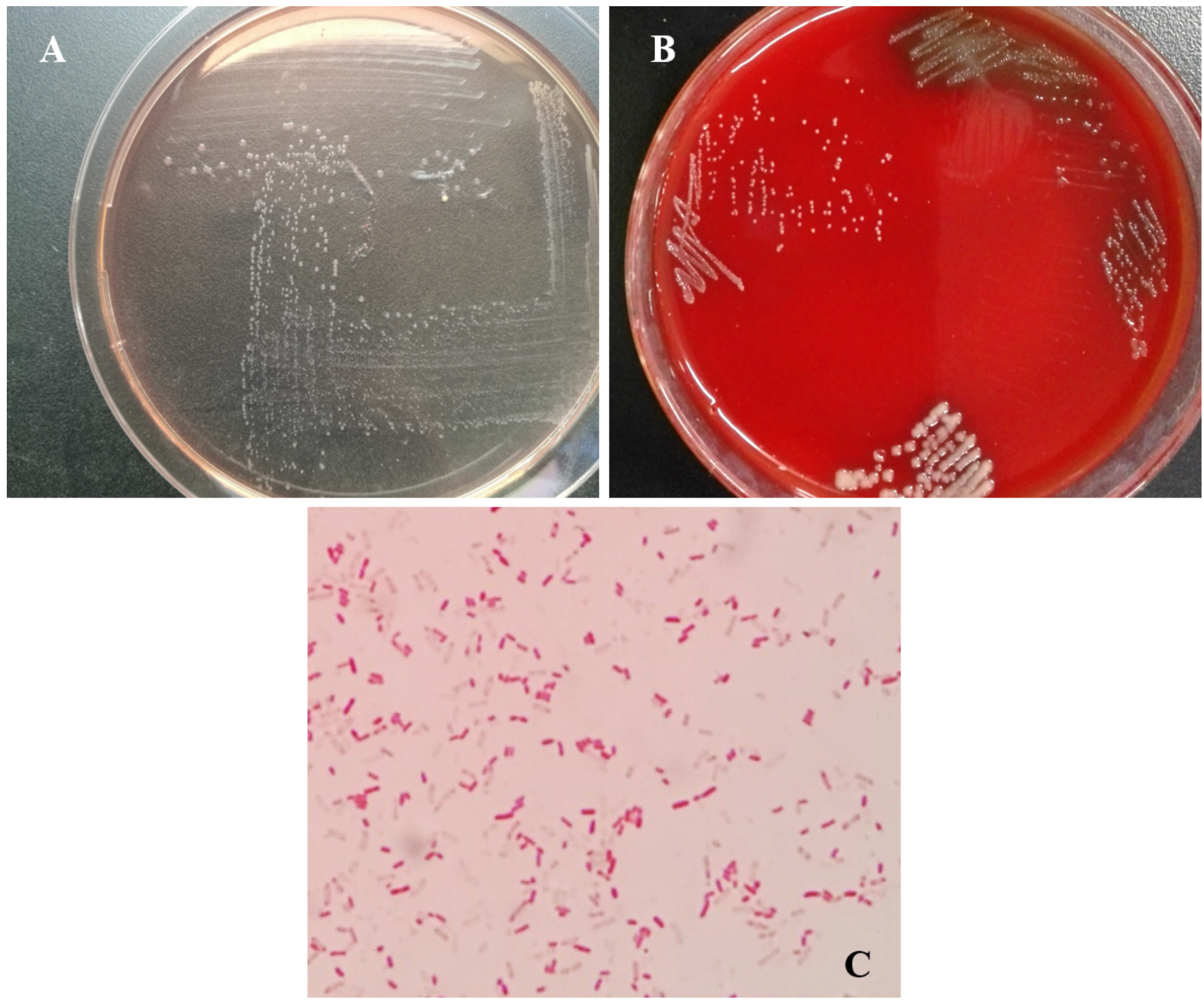

Figure 2

Morphology of Morganella morganii isolates. (A) Colony morphology of M. morganii strains in MacConkey agar. (B) Colony morphology of M. morganii strains in blood agar. (C) Microscopic morphology of M. morganii strains (1000 x). 


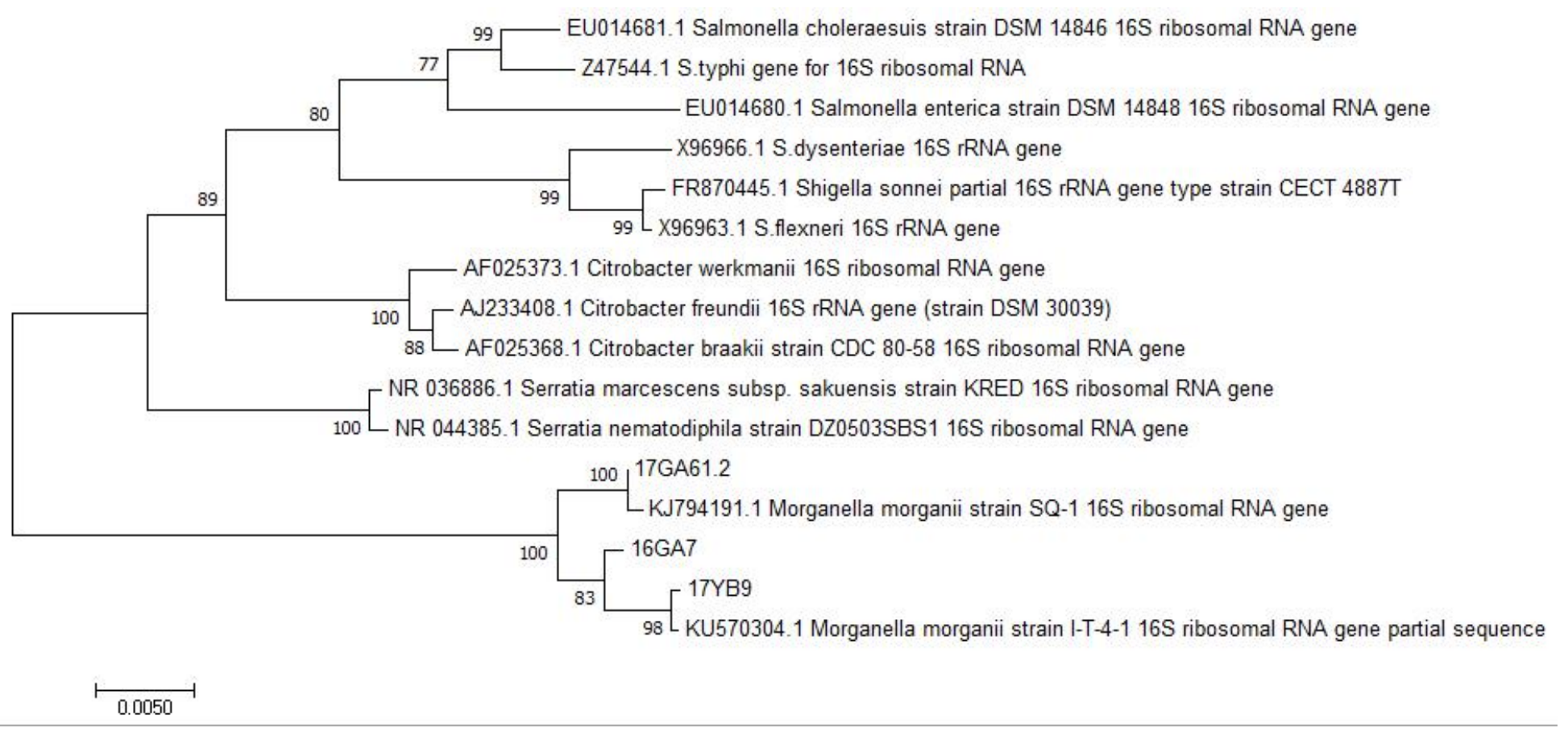

\section{Figure 3}

Phylogenetic tree analysis based on 16SrRNA gene sequences.

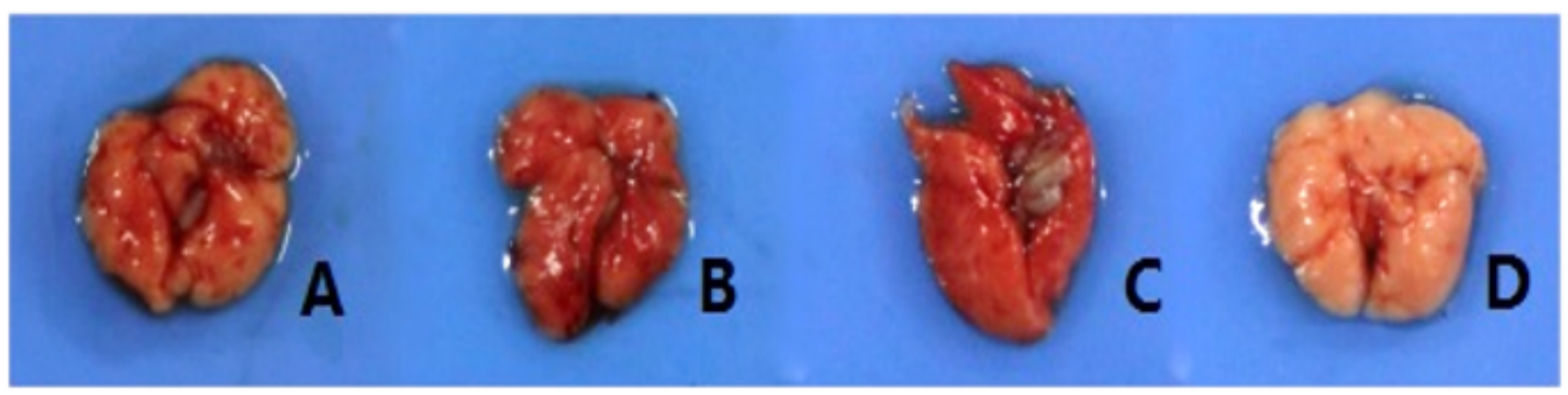

\section{Figure 4}

Representative gross post-mortem pulmonary tissues appearance for each treatment group. (A)16GA7, (B) 17GA61.2, (C)17YB9, (D) control group. 


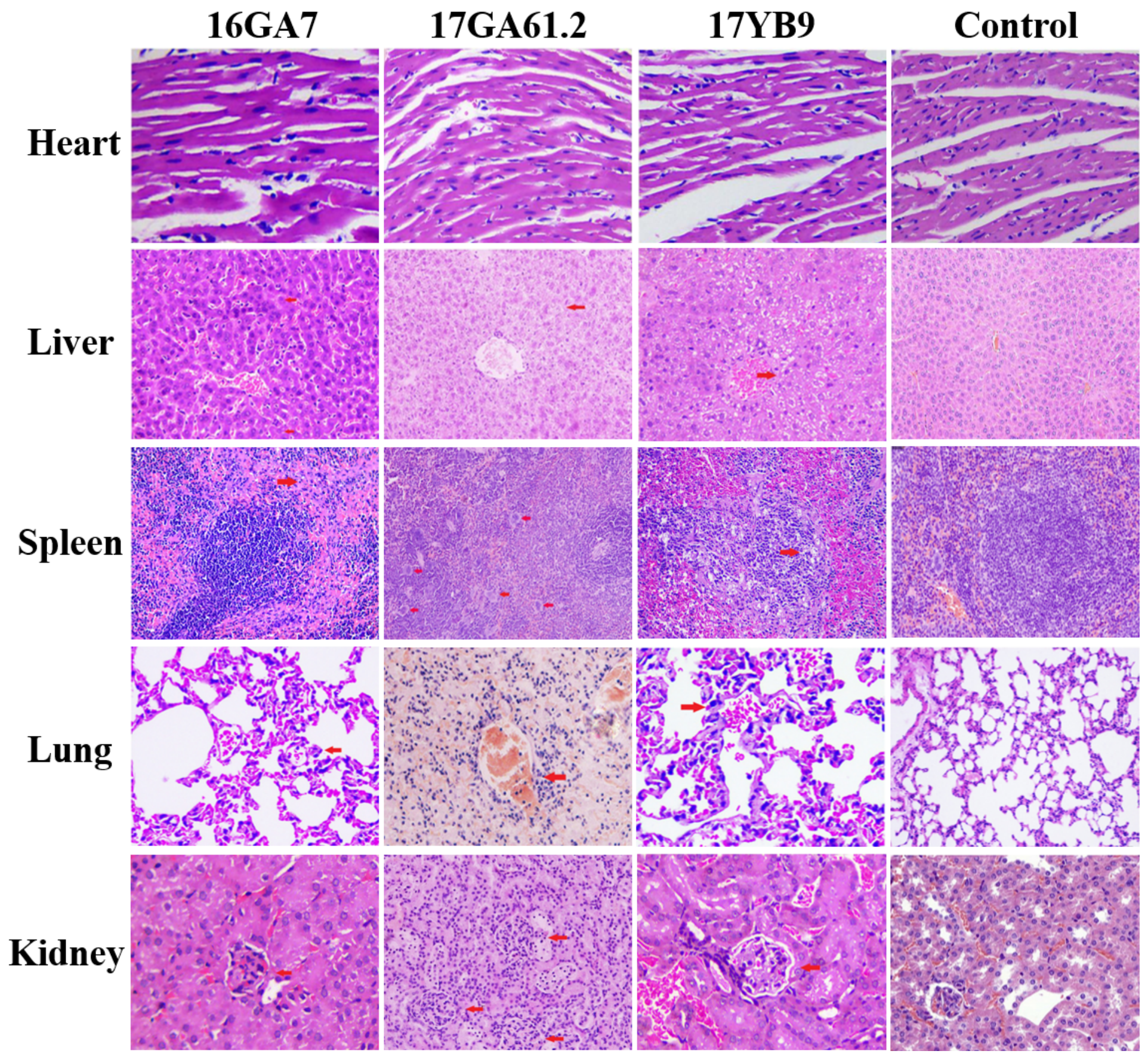

Figure 5

Representative photomicrographs of H\&E-stained heart, liver, spleen, lung, and kidney sections (200x). Arrows for 16GA7, 17GA61.2, and17YB9 photomicrographs identify areas of hepatocyte necrosis. In addition to hepatocyte necrosis, livers from 17YB9 group have vacuolar degeneration. The arrow for the 16GA7 group's spleen indicates splenic congestion, whereas the arrows for the 17GA61.2 group's spleen indicates multinuclear macrophage proliferation, and the arrow for the 17YB9 group's spleen indicates splenic congestion and vacuolar degeneration. The arrows for the 16GA7 and 17YB9 groups in lung tissue indicate areas of pulmonary hemorrhage, alveolar wall thickening and pulmonary inflammatory cell infiltration, and the arrow for the 17GA61.2 group indicates pulmonary hemorrhage. The arrows for the 16GA7, 17GA61.2, and 17YB9 group's kidney photomicrographs indicate narrowing of the renal 
capsular space. In addition to narrowing of renal capsular space, the kidney for the 17YB9 group has vacuolar degeneration.

\section{Supplementary Files}

This is a list of supplementary files associated with this preprint. Click to download.

- NC3RsARRIVEGuidelines2013.pdf 